\title{
A Distributed Genetic Algorithm Improving the Generalization Behavior of Neural Networks
}

\author{
Jürgen Branke, Udo Kohlmorgen, Hartmut Schmeck \\ Institut für Angewandte Informatik \\ und Formale Beschreibungsverfahren \\ Universität Karlsruhe (TH), 76128 Karlsruhe, Germany \\ \{BrankelKohlmorgenlSchmeck\}@aifb.uni-karlsruhe.de
}

\begin{abstract}
Artificial neural networks sometimes generalize poorly to unknown inputs, if they have been trained perfectly on relatively small training sets using standard learning algorithms like e.g. backpropagation. In this paper a distributed genetic algorithm is designed and used to improve the network's generalization capabilities by reducing the number of different weights in the neural network.
\end{abstract}

\section{Introduction}

A difficult and challenging problem in training artificial neural networks is to ensure that they will generalize well to cases they have not been trained on. This is especially true, if the number of training patterns is small compared to the number of independent weights in the network.

Some theoretical results (see Section 2) suggest that networks with few degrees of freedom can be expected to generalize better than those with many degrees of freedom. On the other hand, the network has to be complex enough to be able to represent the training set correctly. To determine an appropriate degree of complexity, several approaches have been suggested that start with training a larger than necessary network and then reduce the complexity e.g. by removing edges or nodes ("pruning" [Ree93]) or by minimizing the number of independent weights ("weight sharing"). Although weight sharing has been used successfully in some applications [CDS89, LeCu89, LWH90, NoHi92], it is not very popular, since it is difficult to decide

- which connections are to be grouped together

- how many groups of connections are needed and

- which weight shall be assigned to each group of connections.

Nowlan and Hinton [NoHi92] developed a method that clusters the weights automatically during the training. The basic idea behind this algorithm is to model the distribution of weight values as a mixture of multiple gaussians. Each gaussian that takes responsibility for a subset of the weights will squeeze those weights together when the variance of the gaussians is shrunk during the process.

In this paper, we report on some experimental results of a different approach to the introduction of weight sharing:

After a network has been trained perfectly on a specific set of training patterns (e.g. by backpropagation), a distributed genetic algorithm is used to find a minimal number of different weights without affecting the network's performance on the 
training set significantly. As expected, this improves the network's performance on a separate test set, i.e. it's generalization ability.

The algorithm is executed on a transputer network. It uses several subpopulations which exchange their best individuals from time to time, but - different from other approaches - the migration does not take place after a predetermined number of generations but after the subpopulations have reached some pre specified performance level.

The problem chosen for testing this approach is the shift-detection-problem that has already been used by Nowlan and Hinton [NoHi92] to compare simple backpropagation, cross-validation, weight decay and soft weight sharing.

The outline of the paper is as follows:

In Section 2, we present some theoretical evidence as to why weight sharing might improve the generalization behavior of neural networks. The main parts of the genetic algorithm are presented in Section 3, followed by a description of the parallelization strategy in Section 4.

Empirical results are reported in Section 5, followed by a summary and some concluding remarks.

\section{Generalization and Weight Sharing}

Artificial neural networks have two basic qualities:

- They can reproduce a set of input-output pairs after having been trained on them.

- They can find some regularities in the training data and use them to generalize to inputs they have not been trained on.

The far more interesting quality definitely is the generalization capability, i.e. the ability that the network produces the desired output given previously unseen input data. This quality can be estimated by measuring the network's performance on a test set disjoint to the training set.

But whereas there exist more or less efficient methods to train a network, it is practically unknown how to build a network that generalizes in the desired way.

An example may illustrate the difficulty: the shift detection problem used in this paper (s. Section 5) has 2048 possible binary input patterns and one binary output, i.e. there exist $2^{2048}$ theoretically possible "rules", each characterized by a distinct truth table. Training the net on 100 training patterns fixes 100 rows of this table, such that $2^{1948}$ rules remain which are consistent with the training set. And we are interested in just one of those rules.

One reason that a network ever has the chance to actually find that rule is that, on one hand, rules in the real world are usually rather simple and, on the other hand, neural nets, depending on their degree of complexity, can only encode rules of a limited complexity.

Intuitively, it would be advantageous to have networks of minimal complexity (such that they can only represent a small fraction of the theoretically possible rules) being complex enough though, to actually represent the desired rule. 
The same idea can be illustrated by an analogy to curve fitting (cf. Figure 1). There, excess parameters result in overfitting. The best interpolation and extrapolation results are obtained with a curve of minimum degree that still represents the "training set" satisfactorily.

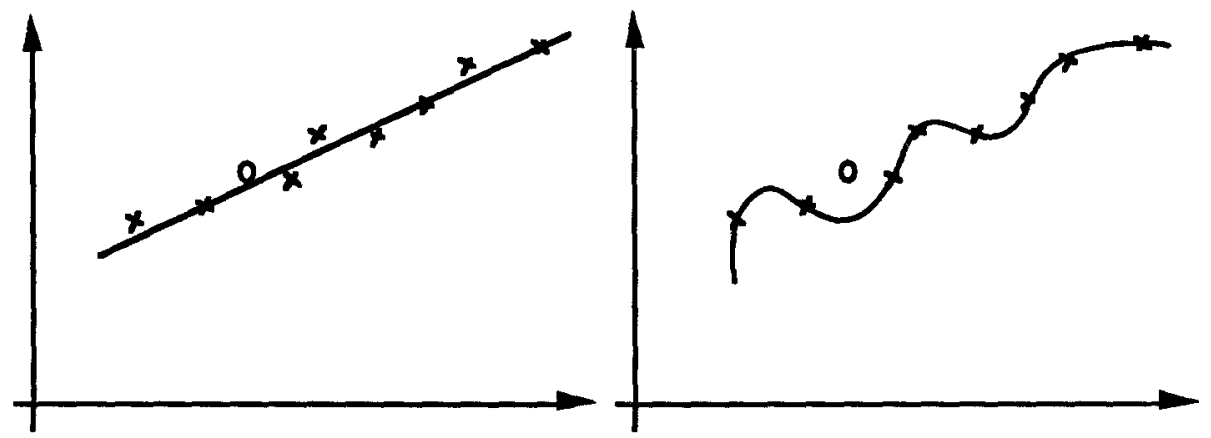

Fig. 1: (Left) Fitting a low-degree polynomial on the training set $(x)$ focuses on the general trend and leads to good interpolation (o) results. (Right) Too many degrees of freedom lead to overfitting and bad generalization (o).

Weight sharing is one way to reduce the network's complexity or degrees of freedom and so seems to be a reasonable approach if one tries to improve the generalization behavior of neural networks.

Apart from this more intuitive reasoning for minimum complexity networks, there exists some theory supporting the use of weight sharing:

The worst case analysis by Vapnik and Chervonenkis [VaCh71] bounds the probability for bad (undesired) generalization dependent on the number of training examples and the complexity of the network. Unfortunately, except for very simple problems, it is hard to exactly determine the VC-dimension, a measure for the network's complexity. Instead, very often the number of independent weights is used as an approximation. For a good introduction to this theory, the reader is referred to [Abu-M89].

Another useful theory in this context is the minimum description length (MDL) principle by Rissanen [Riss78] which states that in the mean, the shortest description of a given sequence of data is the statistical process that generated the data. Therefore networks which can be described with less information are more likely to model the underlying process and also to generalize better. As a network with many equal weights can be described more compact than a network with all the weights independent from each other, this also supports the concept of weight sharing.

\section{Description of the Genetic Algorithm}

Genetic algorithms copy nature by using recombination, mutation, and selection of the fittest in their search for good solutions to optimization problems. For an introduction into genetic algorithms and for some justification for their use, the reader is referred to the literature, for example to the books of Goldberg or Michalewicz [Go189, Mic94]. 


\subsection{General Idea}

Our genetic algorithm starts from a neural network which has been trained perfectly 1 by backpropagation (although any other training method would be acceptable, too). It then tries to partition the ordered set of weights in the network into consecutive groups and to determine new weights assigned to the groups such that the network is still working correctly on the training patterns. More specific, the genetic algorithm determines a set of group borders and group weights. All connections who's weights are lying in the same interval defined by the group borders are regarded as one group and assigned the same group weight (see Figure 2).

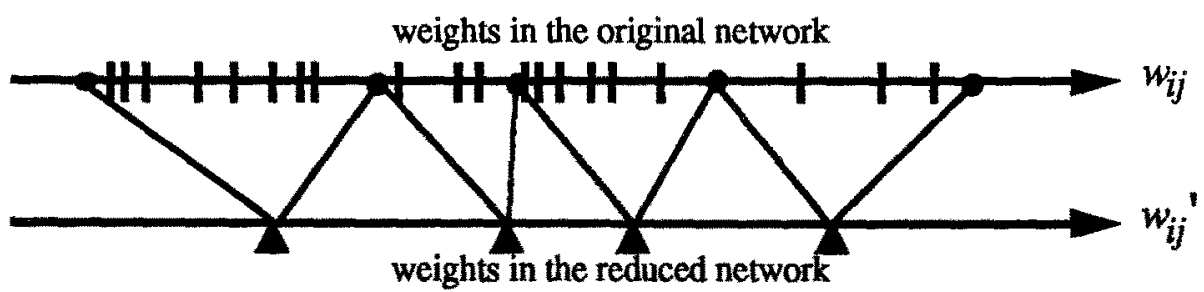

group-borders $\Delta$ group weights

Fig. 2: Reducing a network with 20 weights to a network with 4 weights only.

One may note that the search space in this concept is more or less restricted to the neighborhood of the original trained network. The advantage here is that the search space is small and much information of the original network is preserved. The disadvantage is that many of the best possible solutions may lie outside the search space and will never be found. An alternative model that does not have this limitation is a current target of research at our institute.

\subsection{Representation and Evaluation}

As genetic representation for the individuals, an ascending sequence of group borders and group weights is chosen, forming a blueprint according to which the reduced network may be built (Fig. 3). As it is not known in advance how many groups are necessary, these strings may be of varying length.

To additionally allow the algorithm to completely prune small excess connections, a minimum group weight of 0.0 is implicitly assumed. All connections with an original weight smaller than the smallest group border receive the weight 0.0 and are thereby practically pruned (see dashed connection in Fig. 3).

1 Perfectly here means that the network is capable of correctly classifying $100 \%$ of the training data. Outputs are between -1.0 and +1.0 . Any output $>0.5$ matches 1.0 , any output $<-0.5$ matches -1.0 , i.e. the output is considered correct, if it deviates from the desired output by less than 0.5 . Outputs between -0.5 and +0.5 are alway considered incorrect. 

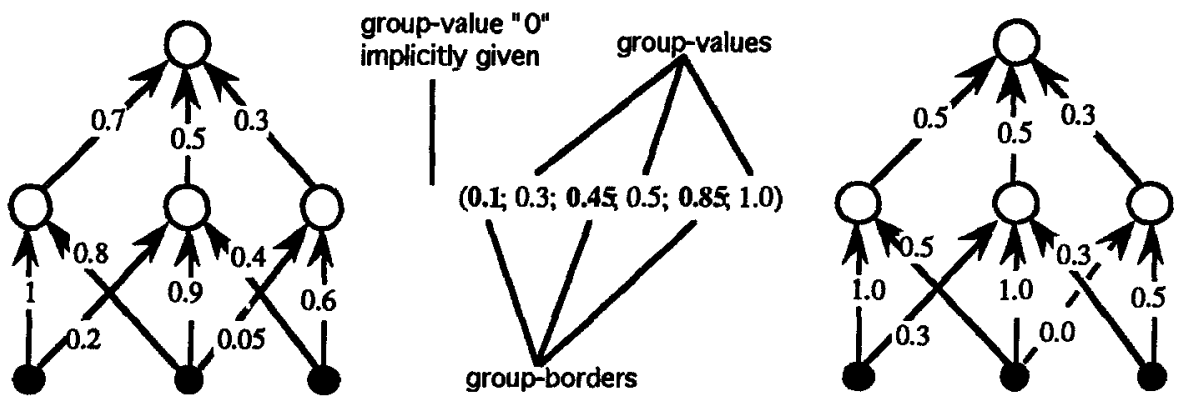

Fig. 3: Transformation of the original network (left) to the reduced network (right) according to the genetic string (middle).

The problem when evaluating the individuals is that it is not possible to measure the generalization capability directly. Thus, following the reasoning of Section 2 , a good strategy may be to search for a network that performs well on the training set (i.e. the set the original net was trained on) while having a minimum number of different weights.

The performance on the training set is determined by building the corresponding reduced network and calculating e.g. the mean squared error. The preference for shorter strings (i.e. a smaller number of different weights) might be included by adding an additional term that penalizes string length. However it could be difficult to find a suitable weighting factor. As an alternative, in our implementation a biased crossover operator is used that occasionally reduces the length of a string (see below). Also, the novel distribution strategy (described in Section 4) contributes to the search for shorter strings.

\subsection{Selection, Crossover, and Mutation}

For production of offspring parents are selected according to roulette wheel selection, i.e. an individual's chance to get selected depends on its relative performance within the population. For recombination of selected parents a single point crossover operator with repair function is used. As crossover point, a position smaller than the length of the shorter parent string is randomly chosen. Whenever the resulting strings are not valid (their values not in ascending order), pairs of values (one group value and one group border) are cut out until the remaining string becomes valid. An example is given in Figure 4.

This crossover operator

- produces valid offspring only

- occasionally introduces shorter strings into the population, i.e. it has a builtin bias towards shorter strings (as desired)

- preserves information from the parents, as group values are mapped to group values and group borders to group borders. 


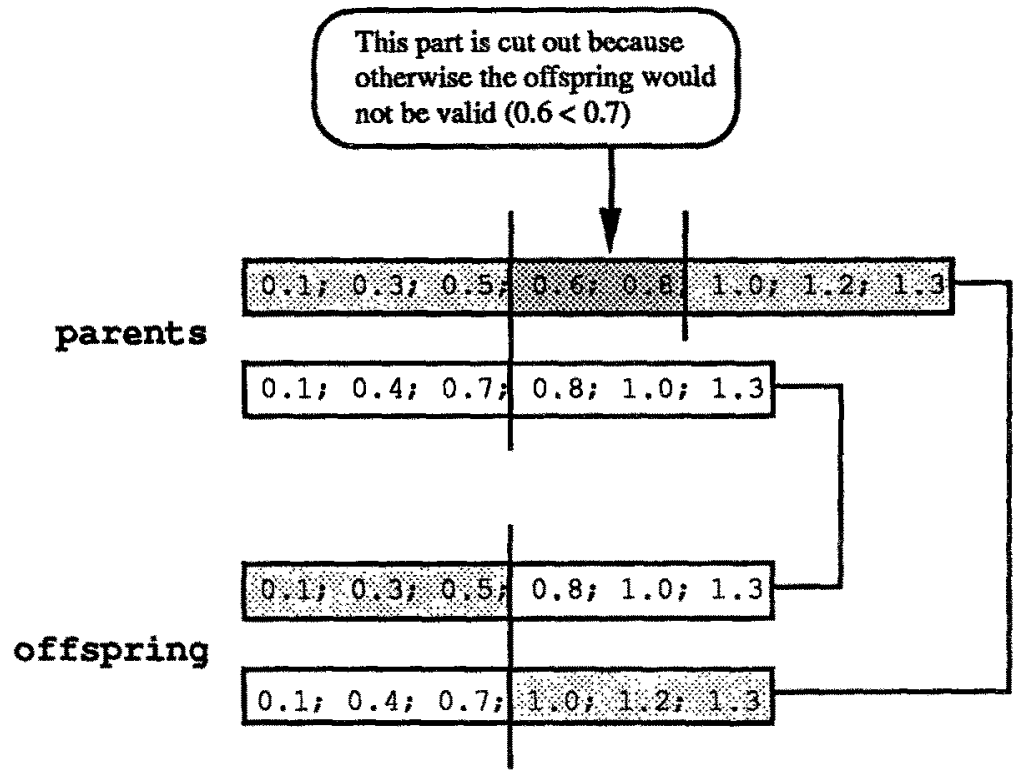

Fig. 4: The crossover operator.

After crossover, each offspring undergoes mutation. Each of the string's positions is subject to mutation with some fixed probability. If a position is chosen for mutation, it is first decided with equal probability whether the value on that position is to be increased or decreased. A new value is then chosen with uniform probability between the old value and the value at the next position to the right or to the left, respectively (see Figure 5). In this way the mutation operator will produce valid strings only.

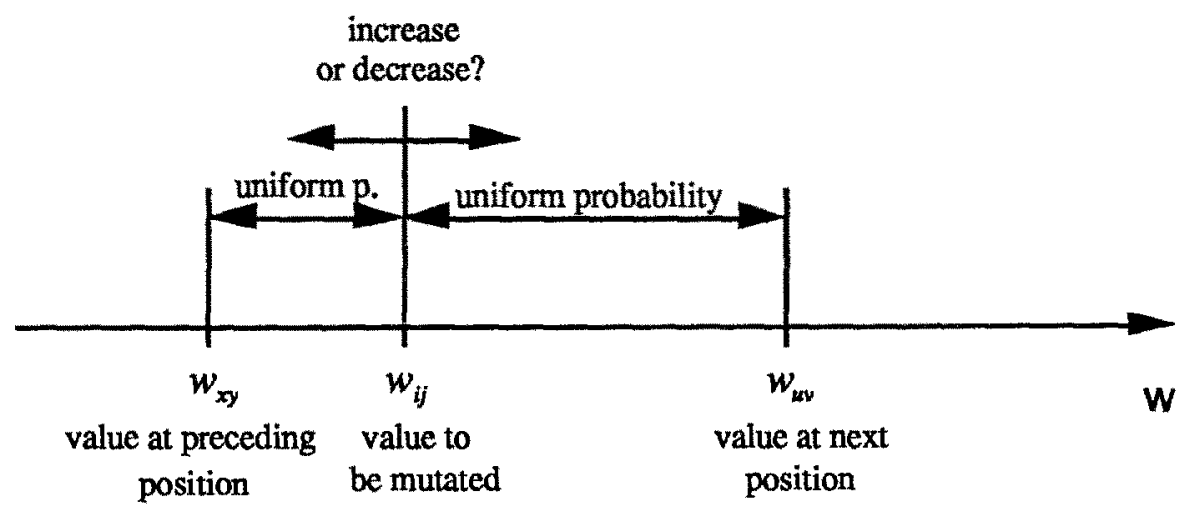

Fig. 5: The mutation operator.

The offspring competes with the individuals of the old population for survival to the next generation. The biggest advantage of this method is that the best individuals 
found so far will always survive and the average performance of the population is increasing monotonically. On the other hand, this bears the risk of drifting towards a uniform population where all individuals are more or less the same.

This risk has been reduced by using large populations and high mutation probabilities.

\section{Distribution Strategy}

Genetic algorithms are highly parallel by nature. Crossover, mutation and evaluation of different individuals can easily be performed in parallel on different processors. The major bottleneck for parallelization is the selection procedure which needs global information to determine the relative performances.

A straightforward parallelization strategy is the so-called farming approach (see e.g. [DMM91]) where a master (farmer) process keeps the global information in form of the entire population, selects the mating partners and distributes those to the processor farm for crossover, mutation and evaluation. The offspring is then returned to the farmer and inserted into the population (Figure 6).

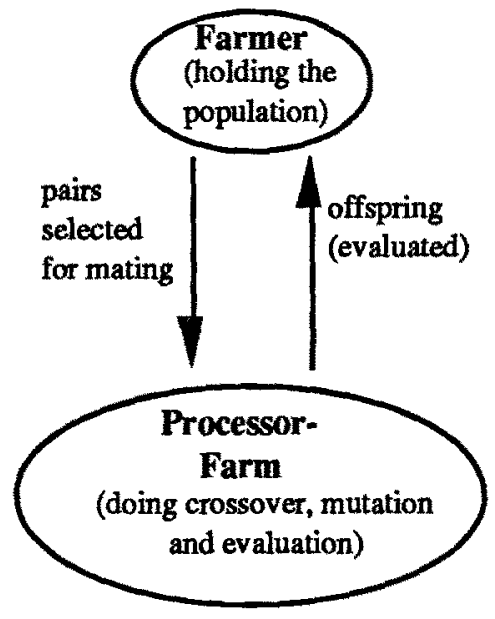

Fig. 6: The Farming Approach.

Although evaluation usually is the most time consuming step, it is obvious that there is a significant communication overhead in this model.

An alternative could be to renounce global information and to distribute the population among several processors, such that every processor runs its own genetic algorithm on a subpopulation. The resulting distributed algorithm is different from the original sequential algorithm, since selection is done locally with respect to the subpopulation only.

This avoids the large communication overhead. Also, by developing the different subpopulations in more or less separate "niches", it very often maintains a larger diversity and avoids premature convergence to a local minimum. A disadvantage, though, is that global information gets lost. This might lead to the processing of locally fit individuals which, according to a global criterion, would be discarded. 
We have chosen an intermediate course insofar as several subpopulations are used, each owning a processor farm (cf. Fig. 8). Occasionally, the subpopulations are allowed to exchange copies of their best individuals with neighboring subpopulations (this is also known as swapping or migration). In this way, the communication overhead is reduced while at the same time the subpopulations share some information.

One problem has been observed with this approach ([WhSt90]): Since the subpopulations evolve at different paces (see Fig. 7) and the migration takes place after some fixed number of generations, it may happen that very 'strong' individuals from a far evolved subpopulation are sent to a 'weak' subpopulation. Also 'weak' individuals could be sent to a 'stronger' subpopulation. In the first case, the immigrated 'strong' individuals may soon dominate the subpopulation, in the latter case, the 'weak' individuals will have no influence on the 'strong' subpopulation and will not enrich its gene base.
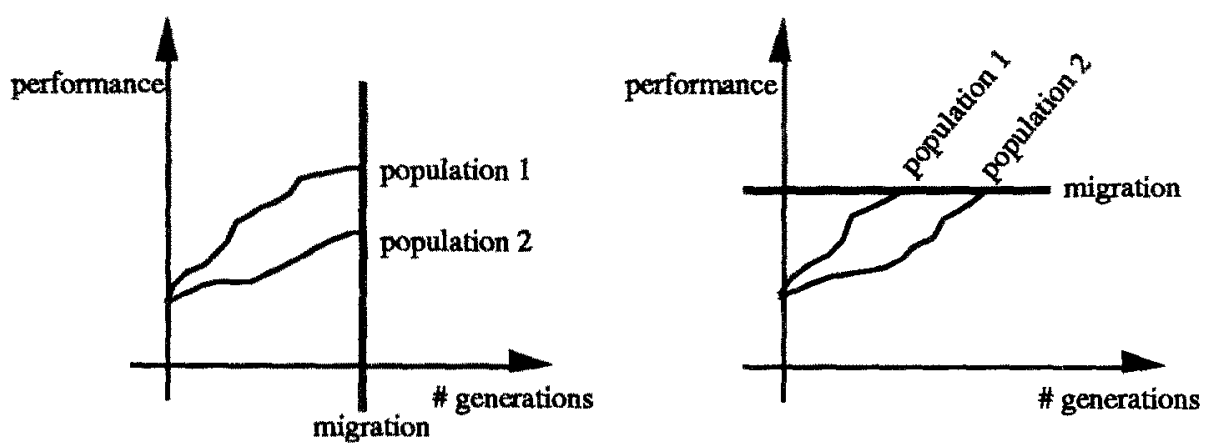

Fig. 7: (Left) If migration takes place after a fixed number of generations, individuals from a far evolved population may soon dominate the weaker population. (Right) If migration is to take place after the populations reached some performance level, different populations will need different times to finish.

None of these cases is desired. In order to avoid the effects, Whitley and Starkweather [WhSt90] suggest to always use rank based selection schemes ${ }^{2}$ that level out the strong differences between individuals.

In this paper, however, a new migration strategy is suggested that allows arbitrary selection schemes.

The basic idea is to perform the migration after the subpopulations have reached some pre specified performance level instead of after a fixed number of generations. A performance measure that has proven successful is for example the average performance over all individuals in the subpopulation. In this way it is assured that the exchanging subpopulations are approximately comparable in performance.

But a new problem arises, namely that the subpopulations will need different times to reach this performance level and thus some populations will have to wait for others. In order to be able to take full advantage of all the available processors anyway, the possibility to lend processors is introduced:

\footnotetext{
2 For a comparison of several selection schemes see for example [Han94], [Bäck94] or [GoDe90].
} 
A farmer (holding one subpopulation) that has reached the performance level may lend its processor farm to still working neighboring subpopulations. The receiving farmer integrates those processors into his farm and may thus process more individuals during the same period of time. As a result, the 'slower' subpopulation usually catches up fairly quickly. When the slower subpopulation reaches the performance level as well ${ }^{3}$, it returns the processors to its owner.

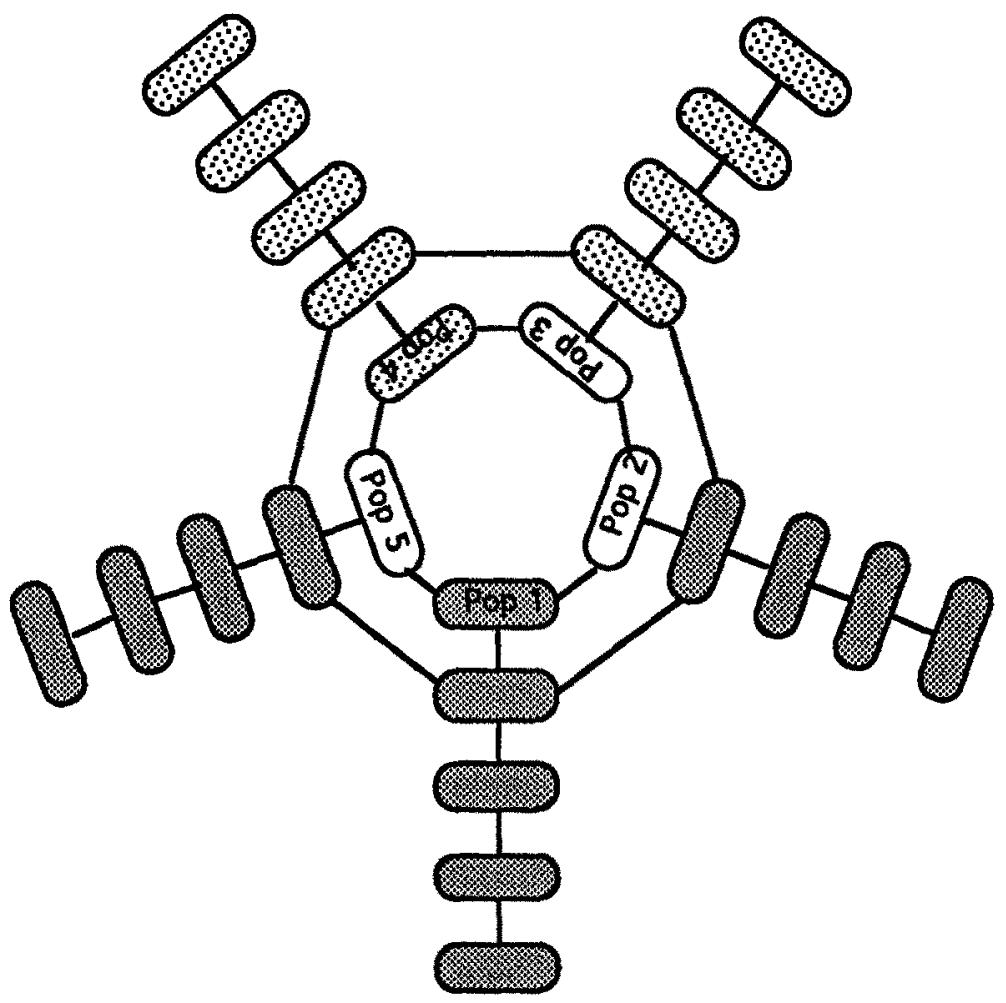

Fig. 8: Hardware topology for 5 subpopulations and 25 processors. The populations 2, 3 and 5 have already reached the performance level and loaned their processor farms to populations 1 and 4.

To realize this algorithm on a 64 transputer-supercluster, a double-ring structure was adopted as shown in Figure 8. The inner ring serves for communication between the farmer processes and for migration. The outer ring is used to integrate borrowed processors. In the simulations described later, 5 populations and 60 processors were used.

3 To make sure that one subpopulation does not block the entire process forever, a maximum number of generations can be specified. If that many generations have elapsed, the migration process is performed no matter what the populations' performances are. 
There are some additional features of the algorithm that make it distinct from standard genetic algorithms and that are especially adapted to the hardware topology.

One is that the number of offspring created during one generation depends on the number of processors in the farm. Each processor is assigned one pair (which could also be 2 or 3 if fewer processors were available) of individuals, performs crossover, mutation and evaluation, and returns one pair of offspring. This guarantees that each processor has about the same amount of workload. As the number of processors available to one subpopulation may vary (due to the borrowing of processors) so may the number of children created in one generation.

A further peculiarity is that the subpopulations were used to level out some 'starting inequalities'. Recall that the algorithm has to deal with genotypes of varying length, depending on how many groups the individual represents. The task is to find a short string (representing few different weights) that still performs reasonably well on the training set. However, by pure statistics, random individuals with many groups are more likely to model the original trained network accurately than are random individuals with fewer groups. This leads to an unfair and undesired 'starting advantage' for longer strings.

This problem is easily overcome by our distribution strategy: The different subpopulations are initialized with different length strings. Therefore, due to the migration strategy, all the subpopulations (and thus all the different length strings) get a chance to reach the first performance level. Only after that competition is introduced slowly by migrating individuals from other subpopulations.

\section{Empirical Results}

The problem chosen for testing our distributed genetic algorithm was the shiftdetection-problem already used by Nowlan and Hinton [NoHi92] to compare simple backpropagation, cross-validation, weight decay and soft weight sharing:

The inputs to the neural network consist of 20 -bit words $(-1 /+1$ representation) such that the second 10 bits correspond to the first 10 bits, but are circularly shifted either to the right or to the left. The network has to detect the direction of the shift. The learning algorithm is applied to a neural network as shown in Figure 9. The desired output of the network is +1 for a left shift and -1 for a right shift.

Out of the 2048 possible patterns, 100 were chosen randomly as training data (and used for the training with backpropagation and for the evaluation in the genetic algorithm), 1300 were used to test the generalization capabilities of the original trained and of the reduced network. The training set was deliberately chosen to be very small to make generalization more difficult.

First, the network was trained until it correctly ${ }^{4}$ classified $100 \%$ of the training data. 10 runs were performed to determine an average percentage of correct answers on the test set of $79 \%$. Then, one network with approximately average performance (78.3\% correct answers on the test set) was chosen and reduced by the proposed genetic algorithm using five subpopulations of 100 individuals each.

4 Correctly again means a deviation from the desired output by less than 0.5 . 


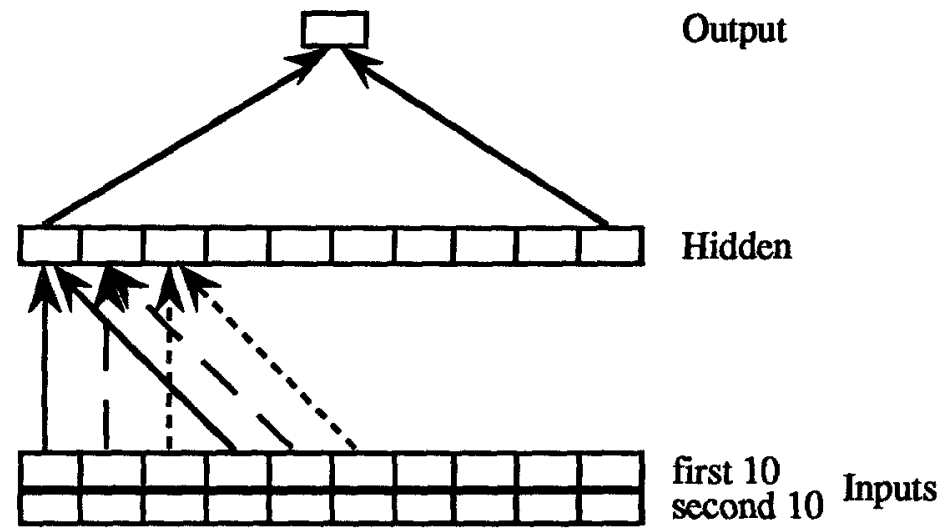

Fig. 9: Shift-detection network used for simulations. Each hidden unit is connected to 8 input units: 4 from the first block of 10 inputs and the corresponding 4 from the second block of 10 inputs. The solid, dashed and dotted lines indicate the group of input units connected to the first, second and third hidden unit, respectively. The output unit is connected to all hidden units. The bias unit is connected to all hidden units and the output unit but omitted in the illustration for clarity (cf. [NoHi92]).

Again, 10 runs were performed with different initial random populations. An average of $84.2 \%$ correct answers on the test set was measured which represents a significant improvement $(\mathrm{p}>99 \%)$. The number of correct answers on the training set decreased slightly to $99.2 \%$.

The final performance level was found after an average of 90 generations ${ }^{5}$. At this stage, the best individuals used 7 or 9 groups of weights.

Table 1 shows a typical result and compares the weight values of the original network with the new values of the reduced network. Although already the original network was not fully connected (101 instead of 211 possible connections), the genetic algorithm pruned another quarter of the connections. It reduced the number of different weights from 101 to only 7 and increased the percentage of correct answers on the test set from $78.3 \%$ to $85.2 \%$.

Further test executions with different parameter settings showed that

- the algorithm works best with a high mutation probability (about $10 \%$ per real value) but is very robust to changes between $5 \%$ and $50 \%$. That is, it still finds equivalent solutions, although it usually takes longer to get them.

- the number of processors available has almost no effect on the solutions found, however, when using half the number of processors it takes approximately twice the time to find the solution.

- compared to the same algorithm using one big population instead of five medium sized subpopulations, the distributed algorithm was able to sustain

5 With the proposed algorithm, not every subpopulation performs the same number of generations. Here, and at all other places in this paper, the maximum over all subpopula'ions is given. 
diversity in string length over a longer period. The results of the distributed algorithm were better, though not significantly $(p>70 \%)$.

- running the algorithm with the proposed new migration strategy and with migration after a fixed number of generations showed no significant differences. One reason may be that the number of exchanged individuals (2) was small compared to the population size $(100)$. Further tests are necessary.

- using the test set for evaluation in the genetic algorithm led to networks with $96 \%$ correct answers on the training set (still used for backpropagation) and $92 \%$ on the test set (used for the GA). In this net, usually $40 \%$ of the connections have been pruned. The experiment shows how tightly the genetic algorithm is restricted to the neighborhood of the original network and that relaxing the restriction might be necessary to yield better results.

- changing the accepted error for an output to be considered correct from 0.5 to 0.3 decreased the performance of the networks that were trained purely by backpropagation to an average of $74.9 \%$ correct answers on the test set. However, running the GA on those nets improved the result again to $84.1 \%$, or approximately to the same performance as with the earlier notion of correctness.

Training with backpropagation until the net perfectly classified the training data with the 0.3 limit, but allowing 0.5 for the GA yielded an average of $87.1 \%$ correct answers on the test set and $99.9 \%$ on the training set. This indicates that it might be possible to further improve the results by using better trained original networks and that the GA will improve the results, no matter how well the original network was trained.

The complete experimental results are documented in [Br94].

The observation that the group weights are often very close to the group borders (cf. Table 1) suggests that the performance might be further improved by relaxing the constraints on the group weights, which always have to lie between group borders due to the chosen representation.

Recent experiments with a representation where the group weights may lie anywhere between the preceding and next group weight (not group border) improved the performance of the same original network to an average of $89.2 \%$ correct classification on the test set and perfect score on the training set. Using a longer trained original network (see last point above) and this less constraint representation even yielded an average of $90.8 \%$ classification accuracy on the test set with relatively low standard deviation $(0.85)$.

When trying to compare the results of this paper to the results reported by Nowlan and Hinton [NoHi92] on the same test problem, the genetic algorithm turns out to work better than cross-validation (that used 1000 additional validation examples) and comparable to Rumelhart's weight-decay, although it did not reach the performance of Nowlan and Hinton's soft weight sharing.

Other than the just mentioned methods, our approach is not restricted to feedforward-networks or differentiable activation functions, and our analysis even suggested ways to further enhance it's performance. 
Tab. 1: Comparison of the original (left) and reduced (right) weights.

\begin{tabular}{|c|c|c|c|}
\hline & -2.296660 & -0.000761 & \multirow{11}{*}{0.0} \\
\hline-1.269776 & \multirow{9}{*}{-1.474317} & 0.009543 & \\
\hline-1.260177 & & 0.021943 & \\
\hline-1.134541 & & 0.027102 & \\
\hline-1.131305 & & 0.040751 & \\
\hline-1.086930 & & 0.051377 & \\
\hline-1.061369 & & 0.060793 & \\
\hline-1.006712 & & 0.068573 & \\
\hline-0.846008 & & 0.072736 & \\
\hline-0.821840 & & 0.096648 & \\
\hline-0.756132 & \multirow{18}{*}{-0.817952} & 0.098216 & \\
\hline-0.679395 & & 0.107132 & \multirow{12}{*}{0.237863} \\
\hline-0.623208 & & 0.119471 & \\
\hline-0.603662 & & 0.135665 & \\
\hline-0.595839 & & 0.143374 & \\
\hline-0.593972 & & 0.154963 & \\
\hline-0.570125 & & 0.155917 & \\
\hline-0.563382 & & 0.180649 & \\
\hline-0.544735 & & 0.183075 & \\
\hline-0.523402 & & 0.230067 & \\
\hline-0.443304 & & 0.261975 & \\
\hline-0.421148 & & 0.265619 & \\
\hline-0.400527 & & 0.273501 & \\
\hline-0.389916 & & 0.306626 & \multirow{22}{*}{0.81792} \\
\hline-0.372866 & & 0.320946 & \\
\hline-0.348698 & & 0.321266 & \\
\hline-0.343247 & & 0.350677 & \\
\hline-0.327538 & & 0.357111 & \\
\hline-0.271977 & \multirow{10}{*}{-0.237863} & 0.390651 & \\
\hline-0.252070 & & 0.391834 & \\
\hline-0.223026 & & 0.418702 & \\
\hline-0.200253 & & 0.443310 & \\
\hline-0.194693 & & 0.450430 & \\
\hline-0.174757 & & 0.460755 & \\
\hline-0.169060 & & 0.506568 & \\
\hline-0.156351 & & 0.521098 & \\
\hline-0.112623 & & 0.537551 & \\
\hline-0.102760 & & 0.545658 & \\
\hline-0.097726 & & 0.555882 & \\
\hline-0.090511 & & 0.622158 & \\
\hline-0.084352 & & 0.651565 & \\
\hline-0.080913 & & 0.689628 & \\
\hline-0.077237 & & 0.693038 & \\
\hline-0.056424 & & 0.732766 & \\
\hline-0.051268 & & 0.783090 & \\
\hline-0.045109 & & 0.846987 & \\
\hline-0.035603 & & 0.880584 & \\
\hline-0.025031 & & 0.908113 & 1.474317 \\
\hline-0.020784 & & 1.010746 & \\
\hline-0.009105 & 0.0 & 1.495531 & \\
\hline-0.004331 & & 1.695201 & 2.296660 \\
\hline
\end{tabular}




\section{Conclusion}

This paper presented a novel approach for improving the generalization capabilities of neural networks: weight sharing on neural networks imposed by a distributed genetic algorithm.

In contrast to conventional distributed algorithms of this kind, here it has been suggested to exchange individuals between subpopulations after they reach some pre specified performance level instead of after some fixed number of generations. This should prevent far evolved individuals from being sent into a weak subpopulation and dominating that subpopulation immediately. Although leading to promising results, this novel migration strategy did not show significantly better performance than the conventional method. However, more experiments on other test problems should be performed before making a final statement on this strategy.

Furthermore, in connection with the performance driven migration strategy, a suitable load balancing strategy has been developed, where far evolved subpopulations can lend processors to slower subpopulations to speed up the evolutionary process.

The presented results show that the proposed algorithm is indeed appropriate to introduce weight sharing on neural networks and that this improves the network's generalization capabilities significantly. In addition, the algorithm turned out to be very robust to parameter changes. The analysis also suggests that even though the algorithm was successful, there is probably still room for improvement.

Future research in this area will include

- further experiments to better understand why and how this approach worked

- development of a new approach that does not limit the search space

- checking whether the algorithm can be helpful in understanding how a neural network actually represents the data and

- evaluating the new migration strategy separately on other problems.

\section{References}

[Abu-M89] Abu-Mostafa, Y. S.: The Vapnik-Chervonenkis Dimension: Information versus Complexity in Learning. Neural Computation 1 (1989), pp. 312-317

[Bäck94] Bäck, T.: Selective Pressure in Evolutionary Algorithms: A Characterization of Selection Mechanisms. Proceedings of the first IEEE conference on Evolutionary Computation, June 1994, Vol. 1, pp.57-62

[Br94] Branke, J.: Weightsharing auf Neuronalen Netzen durch einen parallelen Genetischen Algorithmus. Diplomarbeit, Institut fuer Angewandte Informatik und Formale Beschreibungsverfahren, Universität Karlsruhe, Germany, 1994

[CDS89] LeCun, Y., Denker, J. S. and Solla, S. A., Optimal Brain Damage. In: Advances in Neural Information Processing Systems 1, Denver 1989, D. S. Touretzky, ed., San Mateo, Morgan Kaufmann, pp. 598-605

[DMM91] Dodd, N., Macfairlane, D. and Marland, C.: Optimization of Artificial Neural Network Structure using Genetic Techniques on Multiple Transputers. Transputing 91, P. Welch, D. Stiles, T. Kunii and A. Bakkers, eds., ConferenceProceedings of the World Transputer User Group (WOTUG), Amsterdam, The Netherlands: IOS Press, 1991, pp. 687-700 
[GoDe90] Golderg, D. E. and Deb, K.: A Comparative Analysis of Selection Schemes used in Genetic Algorithms. G. J. E. Rawlins, ed., Foundations of Genetic Algorithms, Morgan kaufman, san Mateo, CA, 1991, pp. 68-93

[Gol89] Goldberg. D. E.: Genetic Algorithms in Search, Optimization, and Machine Learning. Addison-Wesley, 1989

[Han94] Hancock, P. J. B.: An Empirical Comparison of Selection Methods in Evolutionary Algorithms. To appear in: proceedings of the AISB workshop on evolutionary computation, 1994

[LeCu89] LeCun, Y., Generalization and Network Design Strategies. In: Connectionism in Perspective, Pfeifer, R., Schreter, Z., Fogelman, F. and Steels, L., eds., 1989, Zurich, Switzerland. Elsevier, pp. 143-155

[LWH90] Lang, K. J., Waibel, A. H. and Hinton, G. E.: A Time-Delay Neural Network Architecture for Isolated Word Recognition. Neural Networks 3, 1990, pp. 22-43

[Mic94] Michalewicz, Z.: Genetic Algorithms + Data Structures = Evolution Programs. second edition, Springer Verlag Berlin Heidelberg 1994

[NoHi92] Nowlan, S. J. and Hinton, G. E., Simplifying Neural Networks by Soft WeightSharing. In: Neural Computation 4 (1992), pp. 473-493

[Ree93] Reed, R.: Pruning Algorithms - A Survey. In: IEEE Transactions on Neural Networks, Vol.4, No.5 (September 1993), pp. 740-747

[Riss78] Rissanen, J., Modeling by shortest data description. Automatica 14 (1978), pp. 465-471

[SWM90] Starkweather, T., Whitley, D. and Mathias, K.: Optimization using Distributed Genetic Algorithms. In: H.-P. Schwefel, R. Männer (eds.), Parallel ProblemSoving from Nature, Springer, 1990

[VaCh71] Vapnik, V. N. and Chervonenkis, A. Y.: On the Uniform Convergence of Relative Frequencies of Events to Their Probabilities. Theory of Probability and Its Applications 16 (1971), pp. 264-280

[WhSt90] Whitley, D. and Starkweather, T.: GENITOR II: a Distributed Genetic Algorithm. Joumal of Experimental and Theoretical Artificial Intelligence, V. 2(3), (1990), pp. 189-214 